\title{
Peri-implant diseases at a glance - do we have all the answers?
}

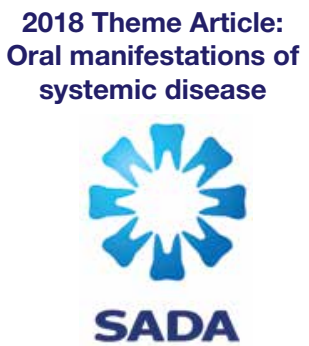

THE SOUTH AFRICAN

SADJ July 2018, Vol 73 no 6 p412 - p416

MM Beetge ${ }^{1}$, VS Todorovic ${ }^{2}$, AW van Zyl. ${ }^{3}$

\section{SUMMARY}

Peri-implant diseases result from chronic inflammatory processes within the tissues surrounding an intra-oral implant. The incidence of the disease is increasing at an alarming rate. Bacterial infections are amongst the several factors that may play roles in the pathogenesis of periimplant diseases. The imbalance between the bacterial biofilm and the host response often leads to tissue destruction. Morphological similarities do exist between the gingiva around teeth and that around the implant, but structural differences in these tissues may possibly influence the host response, and be the reason for the destructive and progressive peri-implant tissue loss. New challenges lie in the diagnosis, treatment, and prevention of peri-implant diseases. This overview focuses on whether the diagnostic criteria used to assess the periodontium can also be applied to peri-implant tissues.

\section{INTRODUCTION}

Whilst dental implants have been in use for more than thirty years to replace missing teeth, it is unfortunate that a new disease has concurrently been created. ${ }^{2}$ The 11th European Workshop in Periodontology recently reported an alarming and increasing prevalence in periimplant disease..$^{2,3}$ The demands of proper diagnosis and treatment and the prevention of peri-implant diseases pose a new challenge in oral health care. ${ }^{4,5}$ Until such time when consensus is reached regarding the array of clinical parameters used to measure and define periimplant diseases, many questions regarding the condition remain, such as: 1) do the anatomical differences between teeth and dental implants influence the host immune response? 2)are periodontal diseases and peri-implant diseases different pathological entities? and 3) can the

1. Michaela M Beetge: $B C h D, M C h D(O M P)$. Department of Periodontics and Oral Medicine, School of Dentistry, University of Pretoria, South Africa.

2. VS Todorovic: DDS, MSc. Department of Oral Surgery, School of Dental Medicine, University of Belgrade, Serbia.

3. Andre W van Zyl: $B C h D, M C h D(O M P)$. Department of Periodontics and Oral Medicine, School of Dentistry, University of Pretoria, South Africa.

Corresponding author

Michaela M Beetge:

Department of Periodontics and Oral Medicine, School of Dentistry, Faculty of Health Sciences, University of Pretoria, PO Box 1266, Pretoria 0001, South Africa. Tel: 012319 2410. E-mail: michaela.beetge@up.ac.za

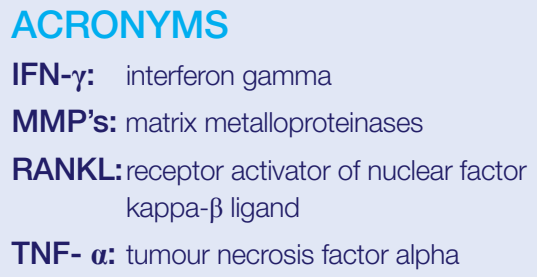

TNF- $\alpha$ : tumour necrosis factor alpha

same diagnostic criteria and indices be used for both periodontal diseases and peri-implant diseases? ${ }^{6}$

The term 'peri-implant disease' was introduced in 2008 at the Sixth European Workshop in Periodontology, and refers to an inflammatory reaction present in the tissues surrounding a functional dental implant. ${ }^{5}$ The imbalance between the bacterial biofilm and the host response is one of the factors that can cause peri-implant tissue loss. ${ }^{7}$

\section{The biofilm}

In health, millions of commensal bacteria are found in the oral cavity. Regular and effective plaque control practices are necessary to prevent the formation of a biofilm. The formation of a biofilm allows periodontopathic organisms to grow and multiply and this in turn activates the host immune response. ${ }^{8}$

In nature, most microorganisms live in biofilms, which protects them from the external environment. The biofilm consists of organized microbial communities, made up of a group of bacterial colonizers that irreversibly attach to a substrate in a wet media, with other colonizers being embedded in their extracellular polysaccharide matrix. The nature of the biofilm allows bacteria to evade the natural defense mechanisms.,9 The outer layers of the subgingival biofilm are in close association with the gingival sulcular epithelium and contain the most pathogenic species. The pathogens secrete endproducts, such as lipopolysaccharides, gingipains and proteases that degrade extracellular matrix proteins and trigger the detachment of fibroblasts from the extracellular matrix. ${ }^{8,10}$ Detachment leads to cell death and subsequent tissue destruction and disease progression. ${ }^{8}$

The biofilm evokes an inflammatory response, the extent of which is dependent on the bacterial load as well as 
on the properties and activation of the immune system. ${ }^{8}$ Commensal oral microbiota exert minimal antigenic reactions and usually exist, when in low numbers, in symbiosis with the host. Pathogens on the other hand, have specific virulence factors that enable them to evade the host inflammatory and immune defense systems, while at the same time triggering chronic inflammatory responses that cause tissue destruction. ${ }^{8}$

Immunopathogenesis and peri-implant tissue loss Adequate blood supply is needed to bring the cells of the immune system to the site of the bacterial insult. The blood supply around dental implants is not as copious as it is around teeth. Ineffective immune responses may lead to the release of a high number of pro-inflammatory cytokines produced by pathogenic bacteria. The proinflammatory cytokines interleukin 1-beta (IL1- $\beta$ ) and tumour necrosis factor alpha (TNF- $\alpha$ ) stimulate fibroblasts, macrophages and epithelial cells to synthesize and release matrix metalloproteinases (MMP's) which in turn cause connective tissue breakdown. ${ }^{8}$ Several mechanisms responsible for bone resorption have been described in the literature,,$^{8,12-14}$ and include:

1. IL1- $\beta$ and TNF- $\alpha$ cytokines impair the coupling of osteoclasts and osteoblasts. Uncoupling leads to disturbances of bone homeostasis, resulting in a dedifferentiation of the osteoblasts, and an increased synthesis of receptor activator of nuclear factor kappa-B ligand (RANKL), which promotes osteoclast activity. ${ }^{13,14}$

2. Dendritic cells are antigen-presenting cells residing in the epithelium and have the ability to trans-differentiate into osteoclasts. ${ }^{12}$ They also take up bacterial antigens and travel to the lymph nodes to present the antigens to naïve T- lymphocytes. Activated T-lymphocytes mainly produce interferon gamma (IFN- $\gamma$ ) and IL-2, but also RANKL. The activated T-lymphocytes play an important role in the humoral immunity by activating B-lymphocytes into antibody-producing plasma cells.

3. Antibodies aggravate bone destruction by stimulating macrophages to produce excessive amounts of proinflammatory cytokines, which in turn stimulates osteoclast recruitment. $^{8}$

Are normal plaque control measures effective for all implant designs?

A dental implant, like a tooth, presents a special challenge to the immune system as it penetrates the basal lamina of the oral epithelium that has its origin from the ectodermal germ layer. If a foreign object interrupts this epithelial integrity, the ideal reaction would expel the object to restore the epithelial continuity. Fortunately, the marginal gingiva is specifically adapted as a zone of tolerance for the penetration of the ectoderm. This soft-tissue attachment around both teeth and dental implants has a similar keratinized oral epithelium that is continuous with the non-keratinized sulcular epithelium. The epithelial and connective tissue apparatus provides a biological seal between the dental implant and the peri-implant soft tissues, or between the natural tooth and the gingival tissues. ${ }^{8,15,16}$ Although the peri-implant soft-tissue barrier has morphological similarities to the gingiva around teeth, structural differences between periodontitis- and periimplant-lesions may influence the host response. ${ }^{17}$ Does the decreased blood supply around dental implants lead to a compromised immune response? Does the compromised immune response allow pathogens to multiply and cause even more tissue destruction? Can findings obtained from using periodontal indices around dental implants be used as reliable indicators of peri-implant tissue health? ${ }^{16}$ These are questions that still require answers.

Periodontal indices used as reliable indicators of peri-implant tissue health

Peri-implant mucositis was first defined as a reversible inflammatory response of the peri-implant soft tissue without any loss of supporting bone around a dental implant. ${ }^{18,19}$ Consensus statements regard peri-implant mucositis and gingivitis as similar entities, with similar clinical findings. The Seventh European Workshop in Periodontology proposed a diagnosis of peri-implant mucositis when gentle probing $(0.25 \mathrm{~N})$ of peri-implant tissues elicits bleeding. ${ }^{17}$ Peri-implantitis differs from periimplant mucositis, in that it includes the loss of supporting bone surrounding a dental implant, as originally defined by the Sixth European Workshop in Periodontology. ${ }^{19}$ During the next Workshop (Seventh), this definition was revised to include changes in the levels of crestal bone over time, as well as bleeding on probing with or without a deepening of probing pocket depth. ${ }^{17}$

Diminished inflammatory signs (redness, heat, pain, swelling and loss of function) in peri-implant disease (Figure 1) bring into question the clinical parameters proposed by several consensus groups. We know that distinct anatomical differences exist between the tissues surrounding teeth and dental implants. Peri-implant connective tissue resembles scar-like tissue as it contains a high number of collagen fibers and low numbers of fibroblasts and vascular structures. The resulting decrease in blood supply, and a lack of the vascular plexus in the peri-implant tissues, make the tissues more susceptible to bacterial insult, while also showing fewer signs of inflammation. ${ }^{20,21}$

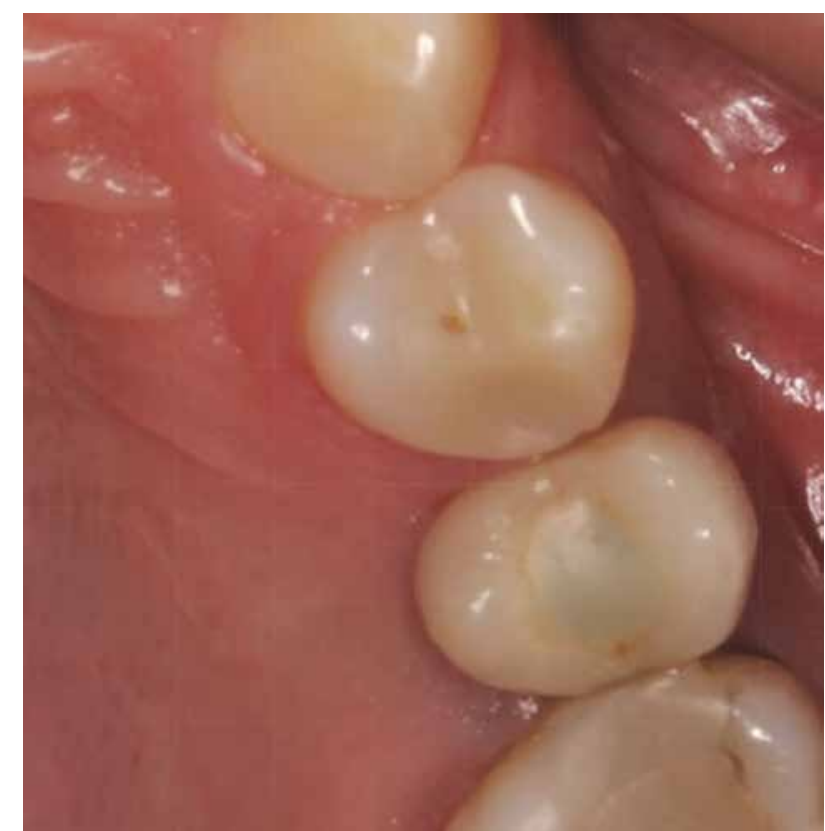

Figure 1: Occlusal view of an implant-supported prosthesis in the 25 area. Lack of inflammation is a predictor for healthy peri-implant tissues. 


\section{a) Clinical probing depth}

Although probing is claimed to be essential for the diagnosis of peri-implant disease, ${ }^{17}$ the reliability of probing depth is currently being questioned. ${ }^{16} \mathrm{~A}$ tooth site is considered to have periodontitis when there is a probing depth of $4 \mathrm{~mm}$ or more, together with fibre attachment loss to the tooth surface. ${ }^{22}$ As result of connective tissue and/or bone destruction, the epithelial attachment migrates apically. ${ }^{8}$ When probing is done around a natural tooth under these circumstances, the tip of the probe penetrates deeper than the average $3 \mathrm{~mm} .{ }^{22}$ There is no connective tissue fibre attachment around a dental implant, therefore, can the diagnostic criteria used to assess the periodontium around teeth also be applied to peri-implant tissues? The poor diagnostic value of probing depths to diagnose peri-implant diseases ${ }^{16}$ contradicts the recommendations currently suggested by Periodontal Societies world-wide. ${ }^{17,19}$ There are no reports in the literature that demonstrate probing depths $>4 \mathrm{~mm}$ as being outside the normal anatomic variation when it comes to dental implants. Deeper probing depths are accepted when dental implants are placed deeper subcrestally, or when mucosal tissues are thicker or more scalloped. ${ }^{16}$ More human studies are needed to determine what significance increasing probing depths over time have on peri-implant tissues. ${ }^{17}$ Additional factors such as implant hardware, surgical technique, or prostheses which may affect probing depth measurements, should also be considered. ${ }^{16}$

\section{b) Bleeding on probing}

While the presence of bleeding on probing is a poor predictor of future disease progression at a periodontal site, the absence of bleeding is a good predictor of future stability. ${ }^{23}$ There are other factors which can cause bleeding upon probing including: excessive probing force, incorrect probe angulation, and increased probe tip diameters. ${ }^{22}$ The vasoconstrictive effects of smoking can mask sites with inflammation and may show less bleeding on probing. According to consensus statements of the Sixth and Seventh European Workshops in Periodontology, the presence of bleeding on probing may be used as a predictor of loss of tissue support when dealing with dental implants. ${ }^{17,19}$ Studies quoted in these consensus statements should be interpreted with caution as some have missing baseline data, for example, crestal bone level changes were not recorded at all times, and incorrect disease definitions were applied. ${ }^{16}$ Thus there is poor evidence to recommend bleeding on probing as a useful clinical parameter for predicting future peri-implant attachment loss. ${ }^{16}$ Bleeding on probing does not always indicate the presence of acute inflammation in peri-implant mucosa, but may rather reflect the nature of the scar tissueimplant interface. ${ }^{16}$

\section{c) Cresal bone level changes}

During the Eighth European Workshop in Periodontology, it was clearly stated that the development of peri-implant disease could only be recognized and followed over subsequent examinations. In the presence of any clinical change, whether bleeding on probing or increased probing depth with or without suppuration, a radiograph might be necessary to evaluate possible bone loss. ${ }^{24}$ This presents a problem when no baseline or previous radiographs are present for comparison. Radiographic evaluation of crestal bone levels over time seems to be the most reliable tool to identify those dental implants undergoing continuous bone loss, ${ }^{16}$ but one should consider the limitations of two-dimensional images as the true nature and extent of tissue destruction are not fully revealed.

Proposed criteria of acceptable bone loss of $1.5 \mathrm{~mm}$ during the first year of loading, and $0.2 \mathrm{~mm}$ annually thereafter, are still widely accepted by the scientific community. ${ }^{16}$ Significant changes in implant treatment protocols have occurred, ranging from using machinedsurfaced implants and mainly restoring the edentulous jaw, to current protocols involving surface-enhanced implants restoring single missing teeth. Many other noninfectious factors may also contribute to early bone loss. The reader is referred to a published article for a more comprehensive review on this topic. ${ }^{25}$ The amount of bone remodeling depends on implant hardware, surgical technique, prosthesis design, and patient factors. ${ }^{16,25}$ The presence or absence of a dental implant-abutment interface that may harbor bacteria plays an important role in peri-implant health. A chronic presence of inflammatory-cell infiltrate is found whenever a micro-gap is present (Figures 2a and 2b). ${ }^{20}$ Histological differences exist between periodontitis- and peri-implantitis lesions.

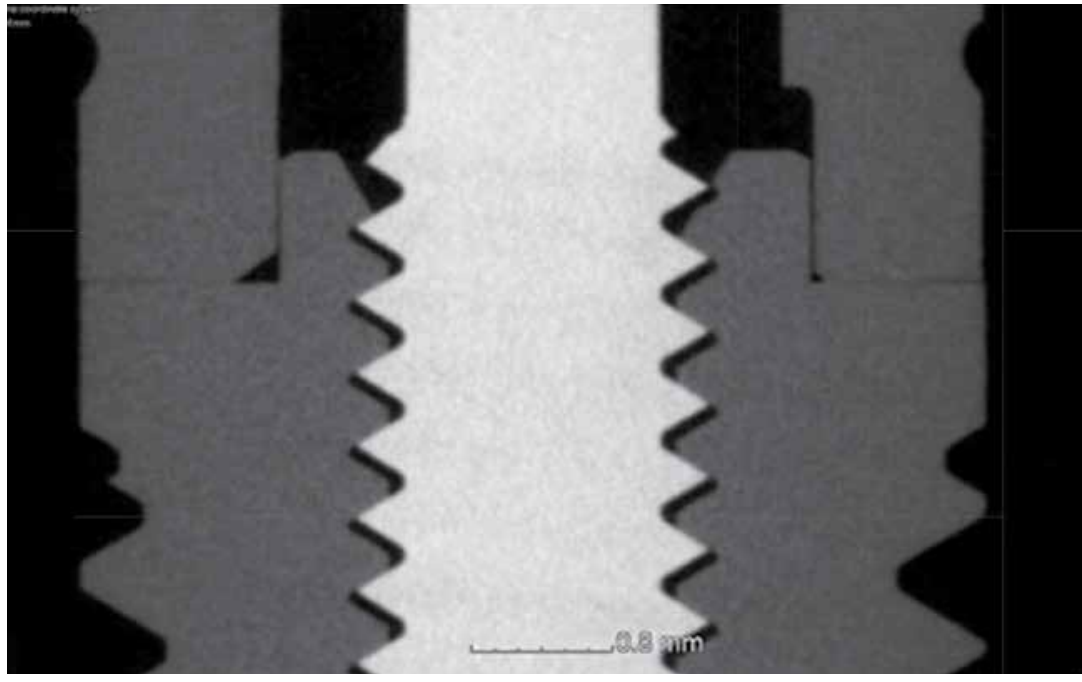

Figure 2a: A micro-focus cone beam tomography image showing the microgap present at the implantabutment interface.

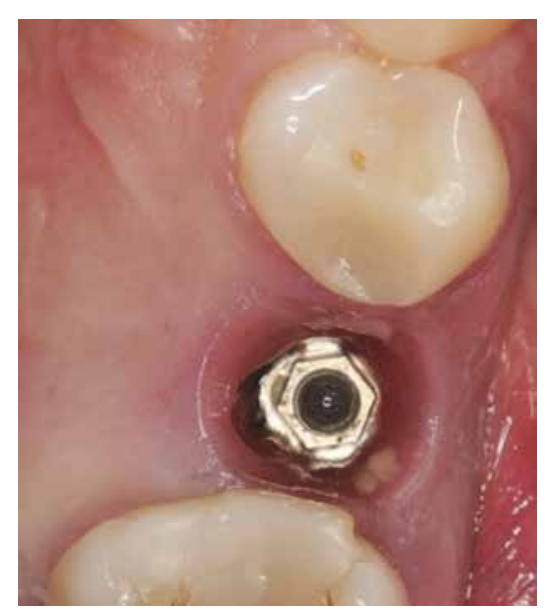

Figure 2b: Extensive plaque accumulation is noticed when the implant crown is removed. The presence of extensive biofilms is commonly encountered in implant systems with large microgaps. 


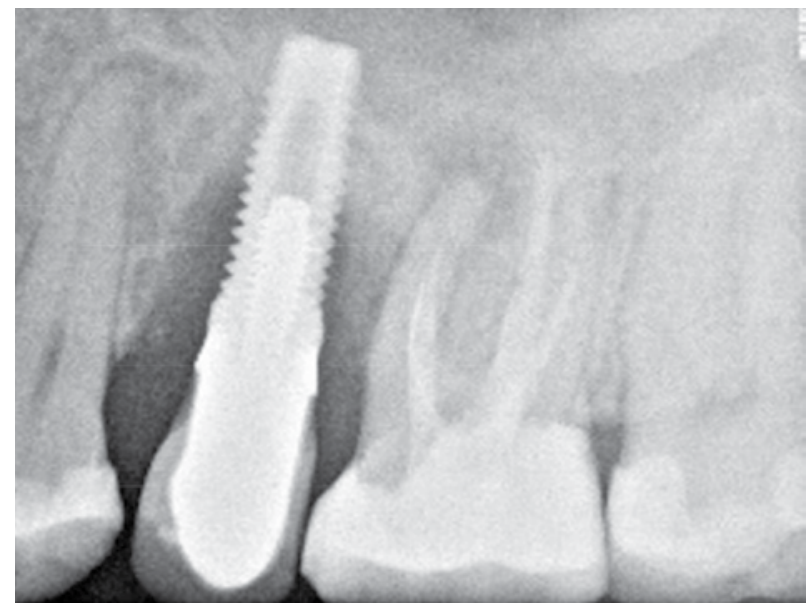

Figure 3: A peri-apical radiograph showing the dental implant in the 25 region. Extensive bone loss as seen around this implant compromises adjacent teeth.

Peri-implantitis lesions are often larger in size and occupy a much greater surface area. These lesions may extend to a position apical to the pocket epithelium, while not being surrounded by non-infiltrated connective tissue as seen in periodontitis lesions. Increased numbers of pathogenic bacteria and a decreased immune response due to anatomical differences between implants and teeth may contribute to a more aggressive and progressive tissue destruction process around implants (Figure 3). ${ }^{26,27}$ Therefore, assumptions that disease within periodontal tissues and peri-implant tissues behave in a similar way regarding etiopathogenesis, diagnosis, classification and treatment, cannot be made. ${ }^{28,29}$

\section{The treatment of peri-implant diseases}

Different etiologies are associated with peri-implant bone loss. Infection within the peri-implant tissues is only one of the causes that may be responsible for peri-implant tissue loss. Peri-implant diseases caused by excess cement or by incorrect surgical protocols can surely not be regarded as a disease process similar to a biofilm-induced periimplantitis. A lack of a standardized classification system to differentiate between peri-implant diseases creates confusion when interpreting results of studies evaluating prevalence and treatment outcomes. ${ }^{4}$ A classification system based on etiology has recently been proposed to differentiate between pathologic (bacterial biofilm) and non-pathologic conditions (residual cement, impacted food debris, buccal implant placement, overheating of the alveolar bone during surgical placement, etc.) that can cause peri-implant diseases. ${ }^{31}$ It is important to correctly diagnose peri-implant disease, as the response to treatment varies widely according to the causative factor. ${ }^{31}$

The goal of peri-implant therapy should also be clearly defined. The use of non-validated end points, such as probing depths of $\geq 4 \mathrm{~mm}$ and bleeding on probing, may be more harmful than beneficial, as this may lead to incorrect diagnoses and unwarranted treatment recommendations. ${ }^{16}$ Peri-implant diseases, irrespective of their cause, are complications that require treatment. Whilst current evidence is not yet sufficient to prove a causal relationship between oral health and general health, the treatment of peri-implant diseases remains important.
Lack of treatment not only affects implant survival, but may also pose risks of systemic inflammation which may influence the overall general health of the patient. ${ }^{32}$

\section{CONCLUSION}

Peri-implant disease is a collective term used to describe chronic inflammatory processes in the tissues surrounding an intra-oral dental implant. There seems to be confusion in the literature regarding etiological factors responsible for the development of peri-implant diseases. A final diagnosis can only be made once the etiological factor has been identified. One cannot apply the same treatment protocol for teeth with periodontal disease to implants with peri-implant disease. Differences in the structural anatomy between teeth and dental implants exist and should be considered whenever treatment is planned. Perhaps the attention should shift away from the diagnosis and treatment of peri-implant diseases, and rather focus more on their prevention. This could be more beneficial, economical, and more successful. Adequate treatment planning and execution may lead to the successful prevention of peri-implant diseases.

\section{References:}

1. Albrektsson T, Zarb G, Worthington P, Eriksson A. The longterm efficacy of currently used dental implants: a review and proposed criteria of success. International Journal of Oral Maxillofacial Implants. 1986;1(1):11-25.

2. Tonetti MS, Chapple IL, Jepsen S, Sanz M. Primary and secondary prevention of periodontal and peri-implant diseases. Journal of Clinical Periodontology. 2015;42(S16).

3. Holland C. Combating peri-implant disease. British Dental Journal 2016;220(2):48-9.

4. Froum SJ, Rosen PS. A proposed classification for periimplantitis. International Journal of Periodontics and Restorative Dentistry 2012;32(5):533-40.

5. Zitzmann N, Berglundh T, Marinello C, Lindhe J. Experimental peri-implant mucositis in man. Journal of Clinical Periodontology 2001;28(6):517-23.

6. Klinge B. Peri-implant marginal bone loss: an academic controversy or a clinical challenge? European Journal of Oral Implantology 2012;5:Suppl S13-9.

7. Esposito M, Hirsch JM, Lekholm U, Thomsen P. Biological factors contributing to failures of osseointegrated oral implants,(II). Etiopathogenesis. European Journal of Oral Sciences 1998;106(3):721-64.

8. Terheyden H, Stadlinger B, Sanz M, Garbe Al, Meyle J. Inflammatory reaction-communication of cells. Clinical Oral Implants Research 2014;25(4):399-407.

9. Kolenbrander PE, Palmer RJ, Periasamy S, Jakubovics NS. Oral multispecies biofilm development and the key role of cellcell distance. Nature Reviews Microbiology 2010;8(7):471-80.

10. Ruggiero S, Cosgarea R, Potempa J, Potempa B, Eick S, Chiquet M. Cleavage of extracellular matrix in periodontitis: gingipains differentially affect cell adhesion activities of fibronectin and tenascin-C. Biochimica et Biophysica Acta (BBA)-Molecular Basis of Disease 2013;1832(4):517-26.

11. Preshaw PM, Taylor JJ. How has research into cytokine interactions and their role in driving immune responses impacted our understanding of periodontitis? Journal of Clinical Periodontology 2011;38(s11):60-84.

12. Alnaeeli M, Park J, Mahamed D, Penninger JM, Teng YTA. Dendritic cells at the osteo-immune interface: implications for inflammation-induced bone loss. Journal of Bone and Mineral Research 2007;22(6):775-80.

13. Graves D, Li J, Cochran D. Inflammation and uncoupling as mechanisms of periodontal bone loss. Journal of Dental Research 2011;90(2):143-53. 
14. Wei S, Kitaura H, Zhou P, Ross FP, Teitelbaum SL. IL-1 mediates TNF-induced osteoclastogenesis. The Journal of Clinical Investigation 2005;115(2):282-90.

15. Bosshardt D, Lang $N$. The junctional epithelium: from health to disease. Journal of Dental Research 2005;84(1):9-20.

16. Coli P, Christiaens V, Sennerby L, Bruyn HD. Reliability of periodontal diagnostic tools for monitoring peri-implant health and disease. Periodontology 2000 2017;73(1):203-17.

17. Lang NP, Berglundh T. Periimplant diseases: where are we now?-Consensus of the Seventh European Workshop on Periodontology. Journal of Clinical Periodontology 2011;38(s11):178-81.

18. Zitzmann NU, Berglundh T. Definition and prevalence of peri-implant diseases. Journal of Clinical Periodontology 2008;35(s8):286-91.

19. Lindhe J, Meyle J. Peri-implant diseases: Consensus Report of the Sixth European Workshop on Periodontology. Journal of Clinical Periodontology 2008;35(s8):282-5.

20. Buser D, Weber HP, Donath K, Fiorellini JP, Paquette DW, Williams RC. Soft tissue reactions to non-submerged unloaded titanium implants in beagle dogs. Journal of Periodontology 1992;63(3):225-35.

21. Degidi M, Artese L, Piattelli A, Scarano A, Shibli JA, Piccirilli M, et al. Histological and immunohistochemical evaluation of the peri-implant soft tissues around machined and acid-etched titanium healing abutments: a prospective randomised study. Clinical Oral Investigations 2012;16(3):857-66.

22. Listgarten M, Mao R, Robinson P. Periodontal probing and the relationship of the probe tip to periodontal tissues. Journal of Periodontology 1976;47(9):511-3.

23. Lang NP, Adler R, Joss A, Nyman S. Absence of bleeding on probing an indicator of periodontal stability. Journal of Clinical Periodontology 1990;17(10):714-21.

24. Sanz M, Chapple IL. Clinical research on peri-implant diseases: consensus report of Working Group Four. Journal of Clinical Periodontology 2012;39(s12):202-6.

25. Tatarakis N, Bashutski J, Wang H-L, Oh T-J. Early implant bone loss: preventable or inevitable? Implant Dentistry 2012;21(5):379-86.

26. Carcuac O, Berglundh T. Composition of human periimplantitis and periodontitis lesions. Journal of Dental Research 2014;93(11):1083-8.

27. Lang NP, Bosshardt DD, Lulic M. Do mucositis lesions around implants differ from gingivitis lesions around teeth? Journal of Clinical Periodontology 2011;38(s11):182-7.

28. Albrektsson T, Buser D, Sennerby L. Crestal bone loss and oral implants. Clinical Implant Dentistry and Related Research 2012;14(6):783-91.

29. Albrektsson T, Dahlin C, Jemt T, Sennerby L, Turri A, Wennerberg $A$. Is marginal bone loss around oral implants the result of a provoked foreign body reaction? Clinical Implant Dentistry and Related Research 2014;16(2):155-65.

30. Norton M. Peri-implant disease: Not the grim reaper? British Dental Journal 2016;220(6):279.

31. Fiorellini JP. A cassification system for peri-implant diseases and conditions. Restorative Dentistry 2016;36:699-705.

32. Jin LJ, Lamster IB, Greenspan JS, Pitts NB, Scully C, Warnakulasuriya S. Global burden of oral diseases: emerging concepts, management and interplay with systemic health. Oral diseases. 2016 Oct;22(7):609-19. PubMed PMID: 26704694. 